\title{
Methods of Assessment of Dynamics of Change of Probability Characteristics of Recognition of Objects in Aerial Reconnaissance
}

\author{
Igor N. Ischuk ${ }^{b}$, Alexey A. Dolgov*a, \\ Andrey M. Filimonov ${ }^{a}$ and Dmitry D. Dmitriev ${ }^{b}$ \\ ${ }^{a}$ Military Education and Research Centre of Military-Air Forces \\ "Military-Air Academy \\ named after Professor N.E. Zhukovsky and Yu.A. Gagarin” \\ 54a Starykh Bolshevikov Str., Voronezh, 394064, Russia \\ ${ }^{b}$ Siberian Federal University \\ 79 Svobodny, Krasnoyarsk, 660041, Russia
}

Received 11.03.2019, received in revised form 20.03.2019, accepted 24.03.2019

The article presents a method of assessing the dynamics of changes in the probabilistic characteristics of recognition and distinction of objects of aerial reconnaissance in the operator and automatic decryption, respectively. The mathematical formulation of the problem of recognition of objects of aerial reconnaissance by infrared images is presented. The results of the evaluation of changes in the probabilistic characteristics of recognition (distinction) of objects of aerial reconnaissance on the results of full-scale experiment.

Keywords: aerial reconnaissance, the probability of successful detection, optimal detection, opticalelectronic system, the unmanned aerial vehicle.

Citation: Ischuk I.N., Dolgov A.A., Filimonov A.M., Dmitriev D.D. Methods of assessment of dynamics of change of probability characteristics of recognition of objects in aerial reconnaissance, J. Sib. Fed. Univ. Eng. technol., 2019, 12(6), 683-693. DOI: 10.17516/1999-494X-0168.

(C) Siberian Federal University. All rights reserved

This work is licensed under a Creative Commons Attribution-NonCommercial 4.0 International License (CC BY-NC 4.0).

* Corresponding author E-mail address: alexdolgov88-08@rambler.ru 


\title{
Методика оценки динамики изменения
}

\section{вероятностных характеристик \\ распознавания объектов воздушной разведки}

\author{
И.Н. Ищук \\ А.М. Филимонов ${ }^{\mathrm{a}}$, Д.Д. Дмитриев ${ }^{\tilde{0}}$ \\ ${ }^{a}$ Военный учебно-научный цеентр Военно-воздушных сил \\ «Военно-воздушная академия \\ имени профессора Н.Е. Жуковского и Ю.А. Гагарина» \\ Россия, 394064, Воронеж, ул. Старых Большевиков, 54 а \\ ${ }^{6}$ Сибирский федеральный университет \\ Россия, 660041, Красноярск, пр. Свободньій, 79
}

В статье представлена методика оценки динамики изменения вероятностных характеристик распознавания и различения объектов воздушной разведки при операторном и автоматическом дешифрировании соответственно. Представлена математическая постановка задачи распознавания объектов воздушной разведки по инфракрасным изображениям. Приведены результаты оценки изменения вероятностных характеристик распознавания (различения) объектов воздушной разведки по результатам натурного эксперимента.

Ключевые слова: воздушная разведка, вероятность распознавания, вероятность оптимального различения, оптико-электронная система, беспилотный летательный аппарат.

\section{Введение}

Появление и развитие комплексов с беспилотными летательными аппаратами (БПЛА) большой продолжительности полета (БПП) с установленными на них современными оптикоэлектронными средствами (ОЭС) привели к значительному росту возможностей воздушной разведки (ВР) наземных целей, ограниченной пространственно-временными, географическими и погодными условиями.

Анализ технических возможностей ОЭС, устанавливаемых на БпЛА БПП, и особенностей их компоновки свидетельствует о необходимости комплексного подхода к сбору и обработке информации, интеграции различных источников информации во взаимосвязанную ОЭС, что, в свою очередь, ведет к повышению возможностей системы, увеличению надежности ее работы, снижению массогабаритных характеристик, что в целом повышает эффективность ведения ВР. При этом основными параметрами, определяющими возможности обнаружения, распознавания и идентификации объекта на изображениях, получаемых с ОЭС БпЛА, являются линейные размеры объекта на изображении, его форма, контраст по отношению к фону, степень неравномерности фона.

Актуальным остается вопрос оценки эффективности применения ОЭС для выполнения задач ВР в различных условиях. Общим критерием эффективности при этом может выступать значение вероятности распознавания объекта на изображении, полученного с ОЭС БпЛА БПП. 
Распознавание объекта воздушной разведки (ОВР) на фоне земной поверхности (ЗП), в качестве которой может выступать, например, грунт (песчаный, травяной и т.д.), вода, бетонные покрытия взлетно-посадочных полос и стоянок, зависит от качества изображения и от его масштаба. Поэтому одним из важнейших параметров процесса дешифрирования является вероятность обнаружения и распознавания изображений ОВР до вида, класса и типа [1]. На величину вероятности обнаружения и распознавания ОВР при ведении ВР влияют различные факторы, например, такие, как высота полета БпЛА с ОЭС ВР, разрешающая способность ОЭС $\mathrm{BP}$, линейные размеры и форма ОВР, тепловой контраст ОВР относительно фона.

\section{Математическая постановка задачи распознавания объектов воздушной разведки по инфракрасным изображениям}

Пусть $U$ - множество ОВР в задаче распознавания. Причем на всем $U$ существует множество $m$ разбиений на подмножества (классы) ОВР $U=\bigcup_{i=1}^{m} \Omega_{i}$.

Каждый ОВР $\omega$ может характеризоваться совокупностью признаков $x_{j} \in X, j=1 \ldots N$, где $X$ - конечномерное метрическое или линейное пространство. Тогда описание ОВР $\omega$ на основе совокупности признаков можно записать в виде

$$
I(\omega)=\left(x_{j}(\omega)\right)+I_{0}, \quad j=1 \ldots N,
$$

где $I_{0}$ - априорные сведения об ОВР.

Исходя из этого формализованная постановка задачи распознавания ОВР на фоне земной поверхности по инфракрасным (ИК) изображениям может быть записана в следующем виде:

$$
\begin{aligned}
& \forall \omega \in U \quad \alpha_{i}=\left(\omega \in \Omega_{i} \mid I(\omega)\right), \quad i=1 \ldots m, \\
& \alpha_{i}=\left\{\begin{array}{lll}
1, & \text { если } \omega \in \Omega_{i}, \\
0, & \text { если } \omega \notin \Omega_{i} .
\end{array}\right.
\end{aligned}
$$

Таким образом, для каждого ОВР $\omega$ необходимо вычислить вектор параметров

$$
\alpha(\omega)=\left(\alpha_{i}(\omega)\right), \quad i=1 \ldots m,
$$

характеризующий принадлежность данного ОВР каждому из существующих классов множества $U$.

\section{Оценка вероятностных характеристик оптимального распознавания объектов воздушной разведки на инфракрасных изображениях}

Множество значений вероятностей распознавания $P_{\text {расп }}$ ОВР на фоне ЗП с использованием зрительного анализатора в зависимости от значения линейного разрешения ОЭС ВР на местности $L$ можно аппроксимировать экспоненциальной зависимостью вида

$$
P_{\text {расп }}=\exp \left(-\left(\frac{B \cdot L^{*}}{l_{\text {пикс }}}\right)^{2}\right)
$$

где $\left(\frac{B \cdot L^{*}}{l_{\text {пикс }}}\right)^{2}-$ показатель ослабления разрешения ОВР на изображении, полученном с ОЭС $\mathrm{BP} ; B=\sqrt{\frac{G\left(R_{\text {впис }}+R_{\text {опис }}\right)}{2 S}}-$ коэффициент распознавания формы ОВР; $G-$ периметр ОВР; $R_{\text {впис, }}$ 
$R_{\text {опис }}$ - радиусы вписанной и описанной окружностей вокруг контура OBP; $S$ - площадь OBP; $L^{*}-$ линейное разрешение на местности при реальном контрасте; $l_{\text {пикс }}=360 \cdot \frac{N \cdot g^{-1}\left(\frac{l_{M}}{2 R}\right)}{\pi \beta_{\Gamma}}-$ максимальный линейный размер ОВР в пикселах; $l_{\mathrm{M}}$ - максимальный линейный размер ОВР, м; $R$ - расстояние до ОВР, м; $N$ - количество элементов приемника излучения в строке матрицы; $\beta_{\Gamma}$ - горизонтальный угол поля зрения (угол захвата) ОЭС, град [1].

Из-за того, что значение линейного разрешения на местности у ОЭС ВР зависит от величины контраста, аппроксимирующую функцию (1) можно представить в виде

$$
P_{\text {расп }}=\exp \left(-\left(\frac{B \cdot L}{l_{\text {пикс } \sqrt{K}}}\right)^{2}\right)
$$

где $L=\frac{R \cdot B}{2 R_{C} f_{\ni}}-$ линейное разрешение на местности [1]; $R$ - расстояние до ОВР; $B$ - коэффициент распознавания формы ОВР; $f_{\ni}=f_{0} \cdot K f=f_{0} \cdot \frac{36}{d_{\Gamma}}-$ фокусное расстояние объектива или датчика в эквиваленте для 35 мм кадра; $f_{0}$ - реальное фокусное расстояние ОЭС ВР; $K_{f}-$ кропфактор - отношение размера ширины матрицы ОЭС ВР к кадру фотопленки тип 135 размером $36 \times 24$ мм; $d_{\Gamma}-$ размер матрицы ОЭС ВР в ширину; $R_{\mathrm{C}}-$ разрешающая способность ОЭС ВР, $\mathrm{MM}^{-1} ; K=\frac{\left|T_{\text {об }}-T_{\phi}\right|}{T_{\text {об }}+T_{\phi}}-$ температурный контраст ОВР и фона; $T_{\text {об }}-$ температура поверхности ОВР; $T_{\phi}$ - температура поверхности фона [2].

При автоматическом дешифрировании задачу распознавания ОВР на фоне ЗП можно рассматривать как задачу оптимального различения двух сигналов, являющуюся частным случаем общей задачи статистической проверки гипотез.

Рассмотрим задачу оценки вероятности оптимального различения двух детерминированных сигналов (ОВР и фон). Для задач различения наиболее обоснованным является применение критерия идеального наблюдателя, минимизирующего суммарную вероятность ошибок 1-го и 2-го рода.

Принимаемое тепловизионным приемником (ТВП) колебание, представляющее собой сумму одного из полезного сигналов (ОВР либо фон) и нормального белого шума (НБШ), в общем виде можно записать выражением

$$
y(n, m, \tau)=\lambda s_{\mathrm{OBP}}(n, m, \tau)+(1-\lambda) s_{\Phi}(n, m, \tau)+w(n, m, \tau), \quad \tau=\text { const },
$$

где $w(n, m, \tau)-$ НБШ; $s_{\text {ОвР }}(n, m, \tau), s_{\Phi}(n, m, \tau)$ - детерминированные сигналы ОВР и фона соответственно; $\lambda=\left\{\begin{array}{lrr}1, & \text { если присутствует } & s_{0 в P}(n, m, \tau) \\ 0, & \text { если присутствует } & s_{\Phi}(n, m, \tau)\end{array}\right.$ - неизвестный параметр.

Априорные вероятности присутствия каждого из сигналов известны. По принятой реализации $y(n, m, \tau)$ необходимо оптимальным образом принять решение, какой из сигналов, $s_{\mathrm{OBP}}(n, m, \tau)$ или $s_{\Phi}(n, m, \tau)$, присутствует на входе ТВП, иначе говоря, найти значение параметра 
$\lambda$. Решение поставленной задачи заключается в статистической проверке двух гипотез: $H_{0}-$ в реализации $y(n, m, \tau)$ присутствует сигнал $s_{\Phi}(n, m, \tau)(\lambda=0)$ и $H_{1}-$ в реализации присутствует сигнал $s_{\mathrm{OBP}}(n, m, \tau)(\lambda=0)$. Априорные вероятности каждой из гипотез $P_{p r}\left(H_{0}\right)$ и $P_{p r}\left(H_{1}\right)=1-P_{p r}\left(H_{0}\right)$ известны.

Апостериорные плотности вероятностей гипотез можно записать с помощью следующих выражений:

$$
\begin{aligned}
& P_{p s}\left(H_{1}\right)=k P_{p r}\left(H_{1}\right) \exp \left[-\frac{1}{N_{0}} \sum_{n=1}^{N} \sum_{m=1}^{M} \sum_{\tau=1}^{T}\left(y_{n, m, \tau}-s_{\mathrm{OBP} n, m, \tau}\right)^{2}\right] \\
& P_{p s}\left(H_{0}\right)=k P_{p r}\left(H_{0}\right) \exp \left[-\frac{1}{N_{0}} \sum_{n=1}^{N} \sum_{m=1}^{M} \sum_{\tau=1}^{T}\left(y_{n, m, \tau}-s_{\Phi n, m, \tau}\right)^{2}\right]
\end{aligned}
$$

или

$$
\begin{aligned}
& P_{p s}\left(H_{1}\right)=k P_{p r}\left(H_{1}\right) \exp \left[-\frac{E_{\mathrm{OBP}}}{N_{0}}+\frac{2}{N_{0}} \sum_{n=1}^{N} \sum_{m=1}^{M} \sum_{\tau=1}^{T} y_{n, m, \tau} S_{\mathrm{OBP} n, m, \tau}\right], \\
& P_{p s}\left(H_{0}\right)=k P_{p r}\left(H_{0}\right) \exp \left[-\frac{E_{\Phi}}{N_{0}}+\frac{2}{N_{0}} \sum_{n=1}^{N} \sum_{m=1}^{M} \sum_{\tau=1}^{T} y_{n, m, \tau} S_{\Phi n, m, \tau}\right],
\end{aligned}
$$

где

$$
\begin{aligned}
& E_{\mathrm{OBP}}=\sum_{n=1}^{N} \sum_{m=1}^{M} \sum_{\tau=1}^{T} s_{\mathrm{OBP}}^{2} \quad n, m, \tau \\
& E_{\Phi}=\sum_{n=1}^{N} \sum_{m=1}^{M} \sum_{\tau=1}^{T} s_{\Phi}^{2} \sum_{n, m, \tau}^{N}=\sigma \varepsilon \sum_{n=1}^{N} \sum_{m=1}^{M} \sum_{m=1}^{M} \sum_{\tau=1}^{T} T_{r \Phi n, m, \tau}^{4} T_{r \mathrm{OBP} n, m, \tau}^{4}
\end{aligned}
$$

$n$ и $m$ - координаты пространственной сетки; $(n, m) \in D=M \cdot N ; \tau \in[1, T]-$ временной интервал наблюдения; $E_{\mathrm{OBP}}$ и $E_{\Phi}-$ энергии сигналов ОВР и фона соответственно.

Согласно критерию идеального наблюдателя принимается гипотеза $H_{1}$, если верно неравенство

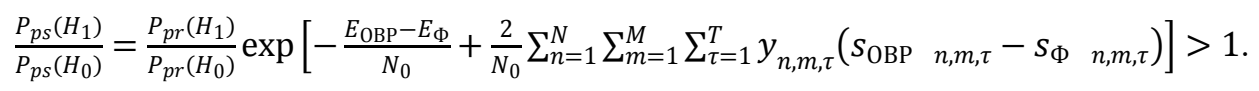

Отсюда следует:

$$
q=\frac{2}{N_{0}} \sum_{n=1}^{N} \sum_{m=1}^{M} \sum_{\tau=1}^{T} y_{n, m, \tau}\left(s_{\mathrm{OBP}} \quad n, m, \tau-s_{\Phi} \quad n, m, \tau\right)>\ln \frac{P_{p r}\left(H_{0}\right)}{P_{p r}\left(H_{1}\right)}+\frac{E_{\mathrm{OBP}}-E_{\Phi}}{N_{0}}=h .
$$

Вероятность общей ошибки

$$
P_{e}=P_{p r}\left(H_{0}\right) P\left(s_{\mathrm{OBP}} \mid s_{\Phi}\right)+P_{p r}\left(H_{1}\right) P\left(s_{\Phi} \mid s_{\mathrm{OBP}}\right),
$$

где $P\left(s_{\mathrm{OBP}} \mid s_{\Phi}\right)=\sum_{h}^{\infty} f_{\Phi}(q)$ - условная вероятность принятия решения о наличии сигнала ОВР, когда в действительности присутствует сигнал фона; $P\left(s_{\Phi} \mid s_{\mathrm{OBP}}\right)=\sum_{-\infty}^{h} f_{\mathrm{OBP}}(q)$ - условная вероятность принятия решения о наличии сигнала фона, когда в действительности присутствует сигнал ОВР. Значение порога оптимального различения можно получить из условия минимума суммарной ошибки $d P_{e} / d h=0$. Таким образом, величина суммарной ошибки:

$$
P_{e}=1-\Phi\left(\sqrt{\frac{E_{\mathrm{OBP}}+E_{\Phi}}{2 N_{0}}\left(1-R_{S}\right)}\right) \text {. }
$$

Отсюда вероятность правильного различения:

$$
\begin{gathered}
P_{\text {пр }}=1-P_{e}=\Phi\left(\sqrt{\frac{E_{0 \mathrm{OP}}+E_{\Phi}}{2 N_{0}}\left(1-R_{S}\right)}\right) . \\
-687-
\end{gathered}
$$




\section{Оценка динамики изменения вероятности оптимального распознавания объектов воздушной разведки по результатам натурного эксперимента}

Оценка вероятностных характеристик распознавания ОВР на изображениях, полученных с ОЭС ВР БпЛА в ИК-диапазоне длин волн, была произведена в ходе натурного суточного эксперимента. Было получено 9 ИК-изображений одного и того же участка земной поверхности с расположенными на ней ОВР с ОЭС комплекса с БпЛА «Гранат-2» с высоты 300 м (рис. 1).

На основе полученных ИК-изображений был произведен расчет пространственного распределения температурных полей исследуемого участка земной поверхности с использованием эталонных точек, в качестве которых были выбраны участки земной поверхности с расположенными на ней объектами, состоящими из материалов с разными теплофизическими свойствами: плита из пенопласта (эталон №1) с теплопроводностью $\lambda=0,026 \ldots 0,09 \frac{\text { Вт }}{\mathrm{M} \cdot К}$, удельной тепло-

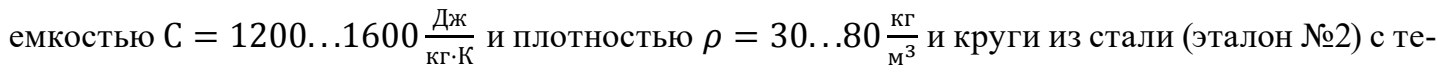
плопроводностью $\lambda=40 \ldots 100 \frac{\text { Вт }}{\mathrm{M} \cdot К}$, удельной теплоемкостью $\mathrm{C}=300 \ldots 500 \frac{\text { Дж }}{\kappa г \cdot К}$ и плотностью $\rho=6000 \ldots 8000 \frac{\kappa г}{\mathrm{~m}^{3}}$ (рис. 2).

Пересчет значений яркостей ИК-изображений в значения радиационных и термодинамических температур производился в соответствии с выражениями:

$$
T_{r}=T_{r 0}+\frac{I \cdot \Delta T_{r \ni}}{\Delta I_{\ni}}
$$
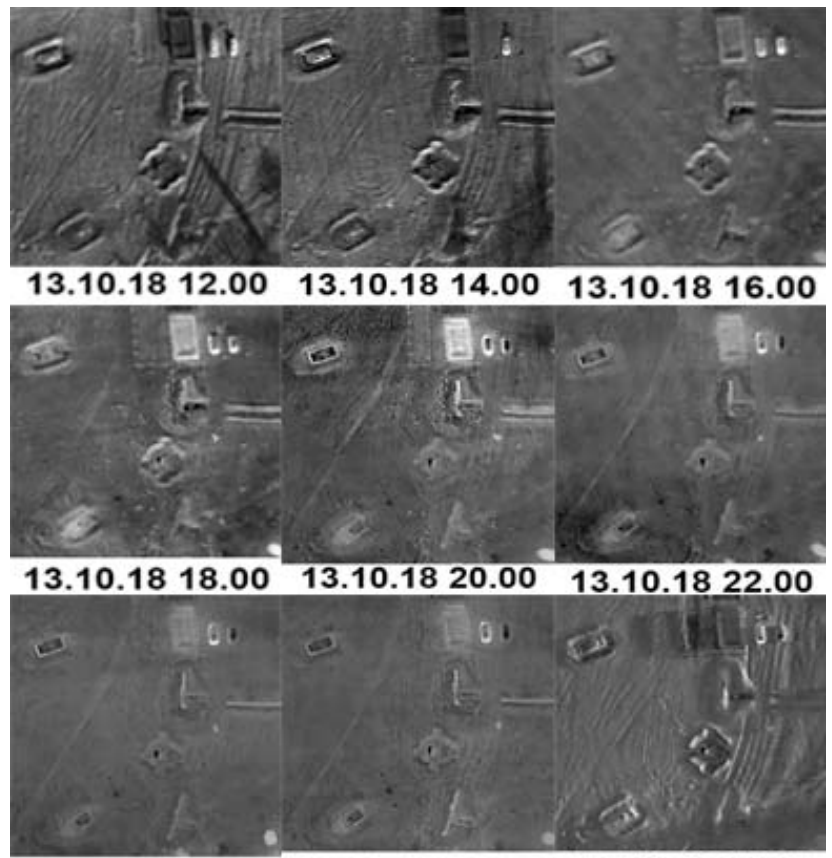

$\mathbf{1 4 . 1 0 . 1 8} \quad 04.00 \quad 14.10 .18 \quad 06.00 \quad 14.10 .1808 .00$

Рис. 1. ИК-изображения района ведения воздушной разведки

Fig. 1. IR images of the area of aerial reconnaissance 


$$
T=\left(\frac{\left|T_{r}^{4}-(1-\varepsilon) T_{A}^{4}\right|}{\varepsilon}\right)^{0.25},
$$

где $T_{r 0}=\left(\varepsilon T_{0}^{4}+(1-\varepsilon) T_{A}^{4}\right)^{0.25} \quad-\quad$ начальная радиационная температура;

$T_{0}=\frac{1}{2}\left(T_{1 \ni}+T_{2 \ni}-\frac{\left(I_{1 \ni}+I_{2 \ni}\right) \Delta T_{\ni}}{\Delta I_{\ni}}\right)-$ начальная термодинамическая температура; $I-$ яркость те-

кущего пиксела ИК-изображения; $\Delta T_{r \ni}=\Delta T_{\ni} \varepsilon^{0.25}=\left|T_{1 \ni}-T_{2 \ni}\right| \varepsilon^{0.25}-$ разность радиационных температур на эталонах 1 и $2 ; \Delta I_{\ni}=\left|I_{1 \ni}-I_{2 \ni}\right|$ - разность яркостей на эталонах 1 и $2 ; \varepsilon-$ коэффициент излучения; $T_{A}$ - температура окружающего воздуха (табл. 1).

В результате преобразований на основе (19) и (20) были получены температурные изображения (термограммы) исследуемого участка земной поверхности (рис. 3).

Для определения оценки вероятностных характеристик распознавания были выбраны два OBP: боевая машина пехоты (БМП) и БМП под маскировочной сетью МКТ-2, а в качестве земной поверхности, являющейся фоном, - участок травяного грунта (рис. 4).

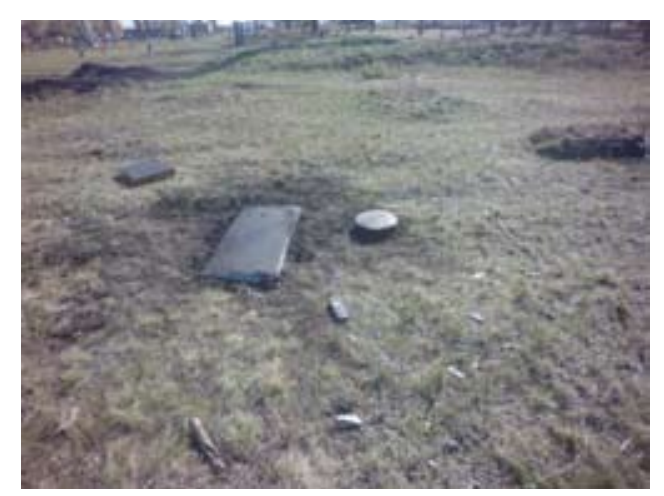

Рис. 2. Расположение эталонных материалов

Fig. 2. Location of reference materials

Таблица 1. Температуры на эталонных материалах и приземного слоя воздуха

Table 1. Temperature reference materials and the ground layer of air

\begin{tabular}{|c|c|c|c|}
\hline Дата, время & $\begin{array}{c}\text { Температура на 1-м } \\
\text { эталоне (пенопласт), } \mathrm{T}_{\text {этl }},{ }^{\circ} \mathrm{C}\end{array}$ & $\begin{array}{c}\text { Температура на 2-м } \\
\text { эталоне (сталь), } \mathrm{T}_{\text {эт2}, ~}{ }^{\circ} \mathrm{C}\end{array}$ & $\begin{array}{c}\text { Температура воздуха } \\
\text { в приземном слое, } \mathrm{T}_{0},{ }^{\circ} \mathrm{C}\end{array}$ \\
\hline 13.10.2018 г. 12.00 & 38.3 & 29.1 & 16.1 \\
\hline 13.10.2018 г. 14.00 & 33.2 & 29.0 & 17.7 \\
\hline 13.10.2018 г. 16.00 & 20.9 & 23.8 & 17.7 \\
\hline 13.10.2018 г. 18.00 & 10.4 & 17.8 & 16.0 \\
\hline 13.10.2018 г. 20.00 & 5.0 & 9.3 & 14.1 \\
\hline 13.10.2018 г. 22.00 & 4.7 & 7.4 & 12.3 \\
\hline 14.10 .2018 г. 04.00 & 2.0 & 4.5 & 8.4 \\
\hline 14.10 .2018 г. 06.00 & 1.0 & 3.5 & 7.2 \\
\hline 14.10.2018 г. 08.00 & 9.8 & 4.1 & 12.5 \\
\hline
\end{tabular}




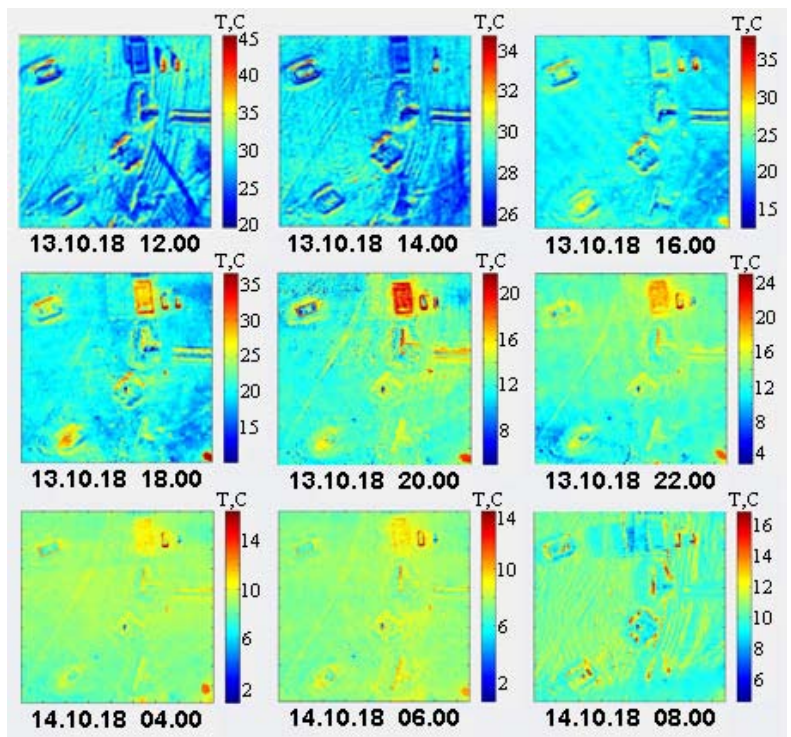

Рис. 3. Термограммы района ведения воздушной разведки

Fig. 3. Thermograms of the area of aerial reconnaissance

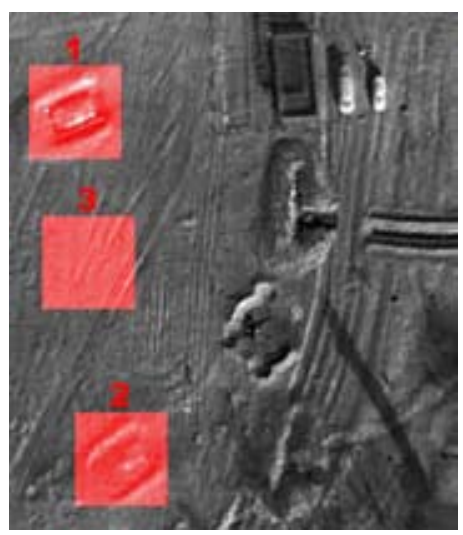

Рис. 4. Район проведения эксперимента (ведения воздушной разведки): 1 - БМП; 2 - БМП под МКТ-2; 3 - травяной грунт

Fig. 4. The area of the experiment (aerial reconnaissance): 1 - BMP; 2 - BMP under MKT-2; 3 - grass soil

Для оценки вероятности распознавания ОВР на фоне земной поверхности при дешифрировании с помощью зрительного анализатора были взяты наиболее контрастирующие участки OBP и фона. При заданных параметрах $L, B$ и $l_{\text {пикс }}$ с помощью выражений (5) и (6) рассчитаем значения температурного контраста ОВР и фона, а также вероятности распознавания ОВР на фоне ЗП (табл. 2).

При автоматическом дешифрировании по температурным распределениям для каждого из заданных ОВР и фона в соответствии с (16), (17) и (18) определим значения вероятности суммарной ошибки $P_{e}$ и вероятности правильного различения $P_{r r}$, а также оптимального порога различения $h_{\text {opt }}$ сигналов ОВР и фона (рис. 5,6$)$. 
Таблица 2. Значения контрастов ОВР и фона и вероятности их распознавания

Table 2. Values of contrasts of objects of air reconnaissance and their background and probability of recognition

\begin{tabular}{|c|c|c|c|c|}
\hline \multirow{2}{*}{ Дата, время } & \multicolumn{2}{|c|}{$\begin{array}{c}\text { БМП } \\
\left(B=1.51 ; l_{\text {пикс }}=55\right)\end{array}$} \\
\cline { 2 - 5 } & \multicolumn{3}{|c|}{$\begin{array}{c}\text { БМП под МКТ-2 } \\
\left(B=1.51 ; l_{\text {пикс }}=55\right)\end{array}$} \\
\cline { 2 - 5 } & $\mathrm{K}$ & $\mathrm{P}_{\text {расп }}$ & $\mathrm{K}$ & $\mathrm{P}_{\text {расп }}$ \\
\hline 13.10 .2018 г. 12.00 & 0.019 & 0.81 & 0.160 & 0.97 \\
\hline 13.10 .2018 г. 14.00 & 0.023 & 0.86 & 0.010 & 0.70 \\
\hline 13.10 .2018 г. 16.00 & 0.075 & 0.95 & 0.090 & 0.96 \\
\hline 13.10 .2018 г. 18.00 & 0.087 & 0.96 & 0.250 & 0.98 \\
\hline 13.10 .2018 г. 20.00 & 0.010 & 0.72 & 0.190 & 0.97 \\
\hline 13.10 .2018 г. 22.00 & 0.052 & 0.93 & 0.020 & 0.95 \\
\hline 14.10 .2018 г. 04.00 & 0.040 & 0.92 & 0.100 & 0.96 \\
\hline 14.10 .2018 г. 06.00 & 0.037 & 0.91 & 0.090 & 0.96 \\
\hline 14.10 .2018 г. 08.00 & 0.135 & 0.97 & & 0.97 \\
\hline
\end{tabular}

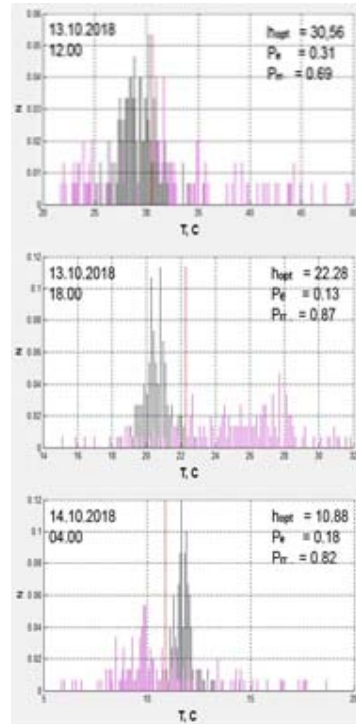

T.C

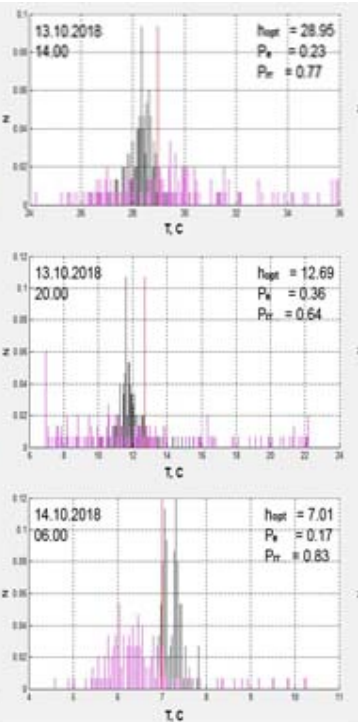

tc

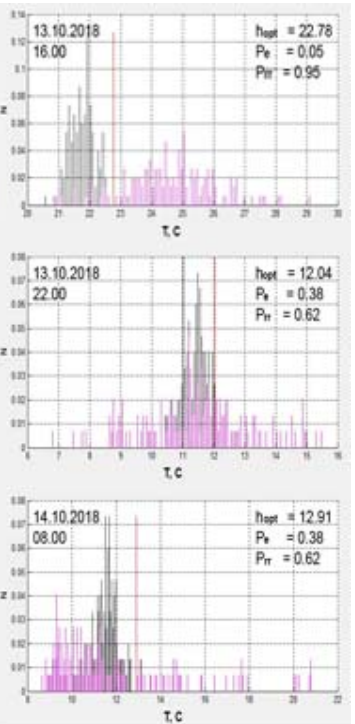

Рис. 5. Гистограммы температурного распределения БМП и травяного грунта

Fig. 5. Histograms of temperature distribution of BMP and grass soil

Графики суточного изменения значений вероятности правильного различения для ОВР типа БМП и БМП под маскировочной сетью МКТ-2 на фоне травяного грунта приведены на рис. 7.

На представленных зависимостях (рис. 8) видно, что значения вероятности распознавания

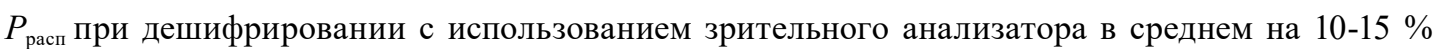
выше значений вероятности оптимального различения $P_{r r}$ при автоматическом дешифрировании. 

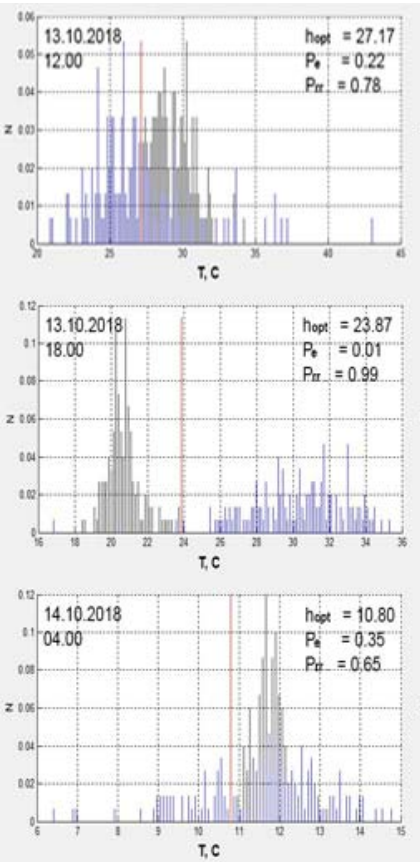
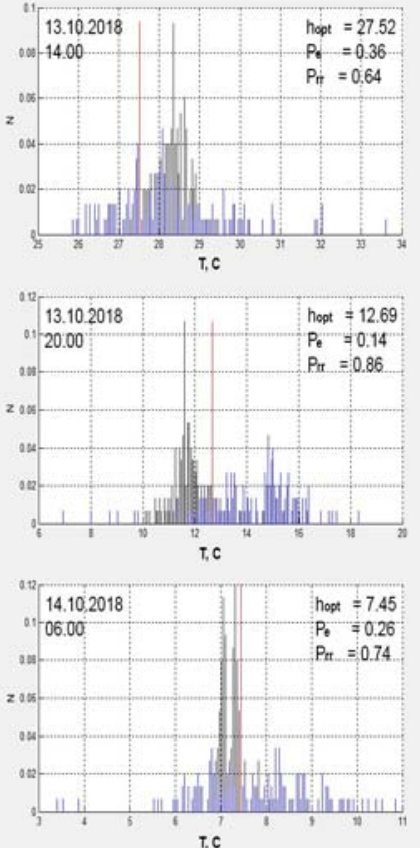

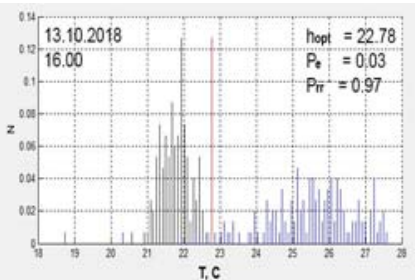

T,C

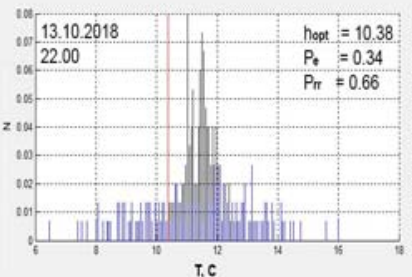

$\mathrm{T}, \mathrm{C}$

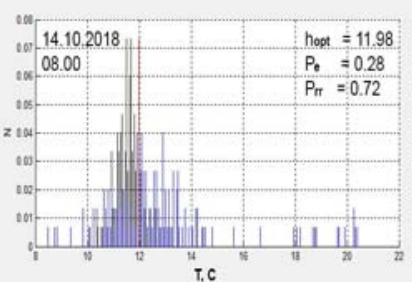

Рис. 6. Гистограммы температурного распределения БМП под МКТ-2 и травяного грунта

Fig. 6. Histograms of the temperature distribution of the BMP under ICB-2 and grass soil

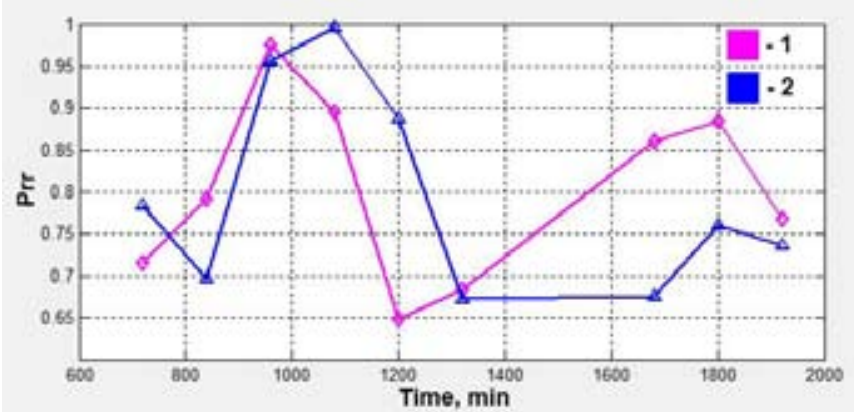

Рис. 7. Динамика изменения вероятности правильного различения ОВР на фоне земной поверхности: 1 - БМП на фоне травяного грунта; 2 - БМП под МКТ-2 на фоне травяного грунта при автоматическом дешифрировании

Fig. 7. Dynamics of change of probability of correct discrimination objects of air reconnaissance on the background of the earth's surface: 1 - BMP on the background of grass-soil; 2 - infantry fighting vehicles under ICB-2 on the background of grass ground with automatic interpretation

\section{Вывод}

Использование предложенной методики оценки динамики изменения вероятностных характеристик распознавания (различения) объектов на фоне земной поверхности, полученных на основе дешифрирования ИК-изображений с применением зрительного и автоматического анализаторов, позволяет определять временные интервалы за сутки, в течение которых вероятность распознавания (различения) ОВР на фоне земной поверхности определенных классов 


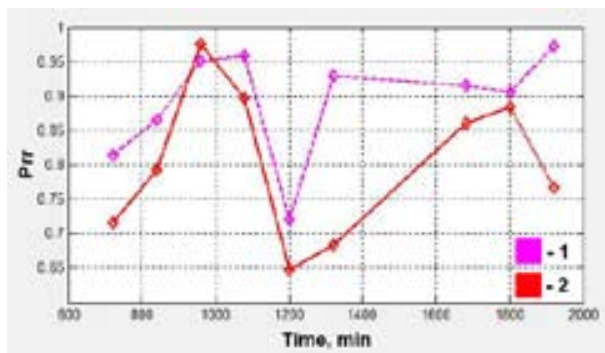

a)

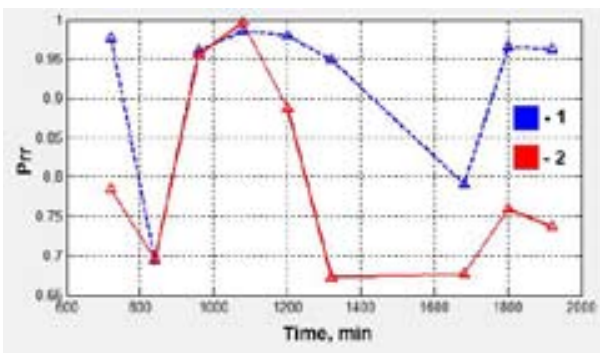

б)

Рис. 8. Динамика изменения вероятности распознавания (правильного различения) ОВР на фоне земной поверхности: а - БМП на фоне травяного грунта; б - БМП под МКТ-2 на фоне травяного грунта при дешифрировании с использованием: 1 - зрительного и 2 - автоматического анализаторов

Fig. 8. Dynamics of changes in the probability of recognition (correct distinction) of objects of air reconnaissance against the earth's surface: a - BMP against the background of grass soil; b - BMP under MKT-2 against the background of grass soil when decoding using: 1 - visual and 2 - automatic analyzers

достигает максимальных или выше заданного порога значений. Это позволяет уменьшить количество вылетов на воздушную разведку и тем самым сократить время её ведения без ухудшения качественных показателей, что в целом ведет к повышению её эффективности.

\section{Работа выполнена при финансовой поддержке РФФИ (грант № 18-08-00053А).}

\section{Список литературы}

[1] Студитский А.С. Исследование и разработка многофункциионального оптикоэлектронного средства наблюдения и разведки. М.: НИИ и МНПО «Спектр», 2013. 112 с. [Studitsky A.S. Research and development of multifunctional optical-electronic surveillance and intelligenc. M.: NII and MSIA "Spectrum”, 2013. 112 p. (in Russian)]

[2] Травникова Н.П. Эффективность визуального поиска. М.: Машиностроение, 1985. 128 c. [Travnikova N.P. Efficiency of visual search. M.: mechanical engineering, 1985, 128 p. (in Russian)]

[3] Алтухов А.И., Гнусарев Н.В., Коршунов Д.С. Прогнозирование качества изображений космических объектов. Научно-технический вестник информационных технологий, механики и оптики, 2013, 3(85) [Altukhov A.I., Gnusarev N.In. Korshunov D.S. Forecasting the quality of images of space objects. Journal Scientific and technical of information technologies, mechanics and optics, 2013, 3(85) (in Russian)]

[4] Веселов Ю.Г., Данилин А.А., Тихонычев В.В. Выбор тест-объекта для оценки разрешающей способности цифровых оптико-электронных систем мониторинга земной поверхности. Наука и образование, 2012, 4 [Veselov Yu.G., Danilin A.A., Tikhonichev V.V. Select the test object for evaluation of the resolution of the digital opto-electronic systems for monitoring the earth's surface. Science and education, 2012, 4 (in Russian)]

[5] Ковалев А.В., Ковалев А.А. Возможности методов неразрушающего контроля. Мup $u$ безопасность, 2007, 2 [Kovalev A.V., Kovalev A.A. Possibilities of non-destructive testing methods. Journal of Peace and security, 2007, 2 (in Russian)]

$$
-693-
$$

